\title{
Trends in Standardization Towards 6G
}

\author{
Nidhi $^{1, *}$, Bahram Khan ${ }^{2}$, Albena Mihovska $^{1}$, Ramjee Prasad $^{1}$ \\ and Fernando J. Velez ${ }^{2}$
}

${ }^{1}$ CGC Research Lab, Department of Business Development and Technology, Aarhus University, Denmark

${ }^{2}$ Instituto de Telecomunicações and Universidade da Beira Interior, Covilhã, Portugal

E-mail:nidhi@btech.au.dk; bahram.khan@lx.it.pt; amihovska@btech.au.dk; ramjee@btech.au.dk; fjv@ubi.pt

${ }^{*}$ Corresponding Author

\author{
Received 02 August 2021; Accepted 07 November 2021; \\ Publication 16 December 2021
}

\begin{abstract}
Mobile networks have always been an indispensable part of a fully connected digital society. The industry and academia have joined hands to develop technologies for the anticipated future wireless communication. The predicted Key Performance Indicators (KPIs) and use cases for the 6G networks have raised the bar high. $6 \mathrm{G}$ networks are developing to provide the required infrastructure for many new devices and services. The $6 \mathrm{G}$ networks are conceptualized to partially inherit 5G technologies and standards but they will open the ground for innovations. This study provides the vision and requirements for beyond 5G (B5G) networks and emphasizes our vision on the required standards to reach a fully functional and interoperable $6 \mathrm{G}$ era in general. We highlight various KPIs and enabling technologies for the B5G networks. In addition, standardization activities and initiatives concerning challenges in the use of spectrum are discussed in detail.
\end{abstract}

Keywords: $6 \mathrm{G}$ networks, spectrum management, standardization development organization, spectrum sharing, terahertz.

Journal of ICT Standardization, Vol. 9_3, 327-348.

doi: 10.13052/jicts2245-800X.932

(C) 2021 River Publishers 


\section{Introduction}

Mobile communication technology has evolved since 1981 from the first generation of mobile communication $(1 \mathrm{G})$ to the upcoming fifth generation (5G) [1]. This evolution shows that a new generation of mobile communication exists every decade and offers new services to the users. Therefore, it is expected that 6G will emerge and come into action around 2030 [2]. Each generation offered enhancements in reliability, data speed, costs, available network functions, power efficacy, security, system performance, latency, network coverage, and services. As a result, disruptive technologies can be seen from each generation, like narrowband in $2 \mathrm{G}$ technology, broadband in $3 \mathrm{G}, 4 \mathrm{G}$ ultra-broadband, wireless world wide web currently in $5 \mathrm{G}$, and expectedly, revolutionary terrestrial wideband in $6 \mathrm{G}$. Besides, there will be enhancements in the bandwidth while data transmission is increased. The 1G began with an analog assembly, which turned to digital communication and circa $25 \mathrm{MHz}$ bandwidth utilization for both $2 \mathrm{G}$ and $3 \mathrm{G}$, while bandwidth increased up to 5 times, to $100 \mathrm{MHz}$ in the case of $4 \mathrm{G}$ networks [3]. 5G use cases are deployed in the $30-300 \mathrm{GHz}$ spectrum bands for wireless communications. On the other hand, $6 \mathrm{G}$ is estimated to demand higher frequency bands utilization, in the range above $300 \mathrm{GHz}$ and up to several $\mathrm{THz}$ [4].

Fast growing data-centric intelligent systems, futuristic scenarios, networks, and applications have imposed challenges to the currently deploying 5G networks [5]. The crucial differentiating characteristic of 5G is low latency, particularly guaranteed latency, that desired deterministic networking to guarantee the end-to-end latency target, with the reliability, required by future use cases. However, $5 \mathrm{G}$ deployment is currently minimal in some typical scenarios, like access in remote areas, e.g, villages or motorways, whose coverage is not satisfactory. Terrestrial $5 \mathrm{G}$ New radio will not support some applications that contain actual driverless vehicles. Therefore, in such environments, satellite communication networks are needed, which can improve the coverage as compared to terrestrial networks. Besides, Unmanned Aerial Vehicles (UAVs) communication networks are essential for quick response in strict and challenging environments, and high-quality communication service can be provided to ships by Maritime communication network. Applications such as Virtual Reality (VR), the mix of VR, high-quality three-dimensional video, and VR and Augmented Reality (AR) will require Tbps, and terahertz or optical frequency bands can be candidate bands.

Although 5G New Radio (NR) is a tremendous evolution step, it will not fulfill all the future network requirements (2030) [6]. Next generation 
networks are likely to assist in several deployment scenarios for different applications, such as coverage from space-air to ground-sea, communications coverage, high-mobility, vehicle to everything, robotic machine, integrating communication, sensing, computing, and high touch VR. 6G is expected to provide nearly $100 \%$ geographical coverage, millisecond geolocation update rate, and sub-centimeter geo-location accuracy to fulfill use cases' requirements and support an enormous amount of data generated by heterogeneous networks, wide bandwidths, large numbers of antennas, and diverse communication scenarios $[5,6]$.

$6 \mathrm{G}$ networks are expected to completely shift the existing communication network to new extreme network capabilities, which cater to the demands of the future data-driven society. The majority of the 5G Key Performance Indicator (KPIs) are valid for the 6G. It is expected that the evolution to $6 \mathrm{Gwill}$ require per link peak throughput that reaches Tbps. Some use cases, like factory automation, will exist, characterized by ultra-high reliability, ultralow latency and high accurate synchronicity, with maximum latency of almost around $1 \mu \mathrm{s}$. In 6G it is expected that in 1 billion bit there will be just one erroneous bit, whilst facilitating outstanding industrial control. Security and privacy are also essential KPIs of 6G. Hyper security high-end user and industrial control will be needed. At the radio interface, a wider bandwidth will be required under the sub- $\mathrm{THz}$ and $\mathrm{THz}$ bands. Therefore, it is essential to understand how the research community is investigating and focusing on the $6 \mathrm{G}$ wireless communication networks.

The use of the spectrum involves many challenges but, at the same, it is an opportunity for future networks. These high-frequency bands will provide a significant role in the overall network. Many exiting IoT scenarios still face cost and battery limitation challenges. However, in 6G, it is expected to provide battery support from the network in some context. On path loss, $\mathrm{H}_{2} \mathrm{O}$ molecular absorption has a significant impact, particularly at a lengthier distance. Penetration through different materials and reflection from the surface are factors to study while classifying the radio spectrum. Although these KPIs must be investigated in more detail, a basic set of the KPIs is shown in Figure 1 [7].

The standards are the key to smoothening the timely journey towards $6 \mathrm{G}$ networks. Deciding whether they are open or not helps to have a unified approach throughout the pre-development and development phase. Additionally, it helps to join forces against obstacles en route and accelerate innovation. Many standardization bodies are working in similar directions, in similar or different areas. There are huge overlaps and intersections; thus, 


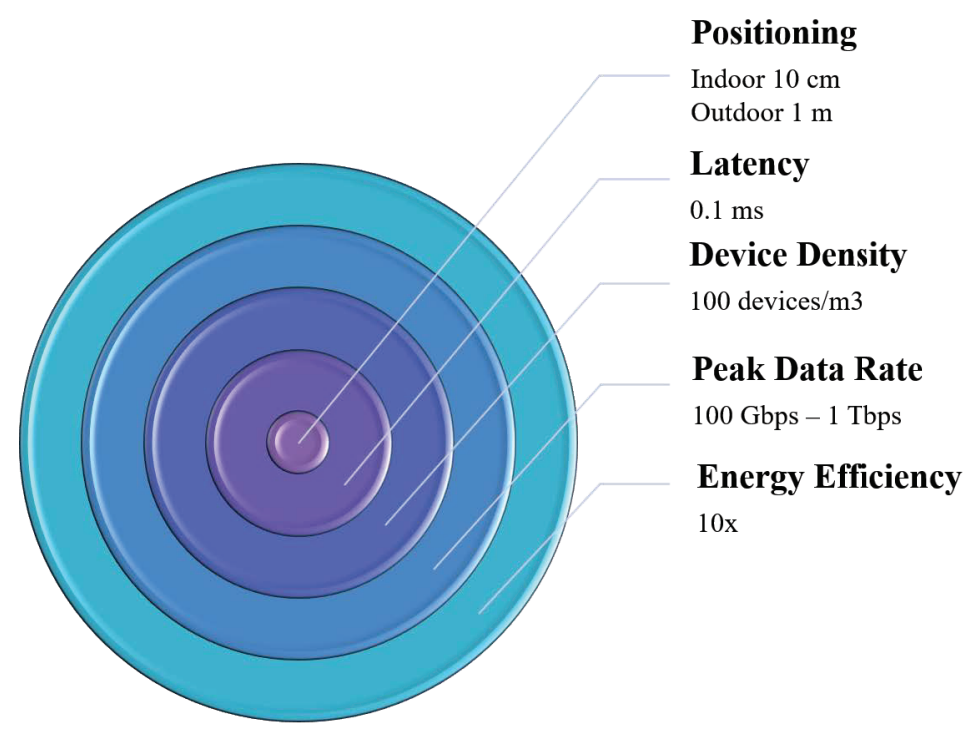

Figure 1 Key performance indicators for $6 \mathrm{G}$.

it is essential to facilitate the idea of cross-research. Furthermore, these standard bodies work to identify the right time to initiate actions concerning pre-standards or full standards activities.

This paper aims to draw a complete picture of $6 \mathrm{G}$ Networks from a standardization perspective. First, we define the concept of a standardization body and how it operates at various levels. Then, the paper contributes to the $6 \mathrm{G}$ expectations, services, identified KPIs, and challenges. The remaining paper is outlined as follows: Section 2 addresses the Spectrum Management in $6 \mathrm{G}$, Section 3 describes various $6 \mathrm{G}$ use cases, and Section 4 addresses $6 \mathrm{G}$ enabling technologies. Section 5 describes the role of standards and relevant standardization bodies. Sections 6 and 7 addresses the spectrum requirements in $6 \mathrm{G}$ and describes standardization activities in detail. Finally, conclusions are drawn in Section 8.

\section{Spectrum Management}

Spectrum management efficiently handles available frequency bands and has taken several arrangements and tactics in the history of mobile communication networks [8]. It comprises the spectrum allocation, which has been based on an administrative approach, common unlicensed mechanisms, and 
an allocation scheme, depending on the market. In the literature, the available details of $6 \mathrm{G}$ communication networks and their spectrum are limited, as research is still in its early stages. It is expected that $6 \mathrm{G}$ will be deployed in the Terahertz (THz) frequency bands range aimed at mobile communications [9]. Spectrum bands presently under $2 \mathrm{G} / 3 \mathrm{G} / 4 \mathrm{G} / 5 \mathrm{G}$ in different countries need to be available for $6 \mathrm{G}$ [10]. The $6 \mathrm{G}$ spectrum bands will also be allocated in frequencies higher than before, leading to various spectrum management approaches that imply distinguishing the higher, mid, and lower bands.

Spectrum management of $6 \mathrm{G}$ frequency bands is challenging and will combine sensing with communications. The inclusion of Artificial Intelligence (AI) in spectrum access decisions will open the door for full dynamic operation. $6 \mathrm{G}$ networks will sense the environment of the radio and will adapt it in real scenarios $[11,12]$.

Figure 2 shows the $5 \mathrm{G}$ and $6 \mathrm{G}$ available frequency bands and presents their basic properties. It explains that the increase in free space loss is slight when going into the $\mathrm{THz}$ region, and from $30 \mathrm{GHz}$ onward. With constant antenna area, the free space loss is compensated by the increase in the antenna gain. Relatively the free space loss, the downside of higher frequencies is the rise of the complexity and parallelism in RF hardware. The underlying reduced beam width creates signal acquisition and beam tracking problems in different mobile applications. Different IoT devices' costs, ranges, battery limitations will not quickly scale with the evolution to higher frequencies. The THz spectrum must be managed in terms of path loss and according to the sub-bands reflection properties to optimize resource reuse. In those scenarios that support multiple applications, the overlap of different sub-channels must be prevented by carefully performing frequency planning. Figure 2 shows that whil frequency bands up to $3 \mathrm{GHz}$ support up to $10 \mathrm{~km}$ link distances, coverage ranges in upper-frequency bands will be significantly less [13].

\section{6G Use Cases}

The KPIs identified for the 2nd phase of $5 \mathrm{G}$ hold importance in $6 \mathrm{G}$ networks and are slightly modified based on new $6 \mathrm{G}$ network requirements. A rich blend of use cases that explore 6G features can be identified in [15]. The proposed $6 \mathrm{G}$ use cases are concentrated on positioning, latency, device density, peak data rate, and energy efficiency. It ranges from human twin to manufacturing units (smart factory plus). The human digital twin requires low latency and peak data rate, while the smart factory additionally needs security 


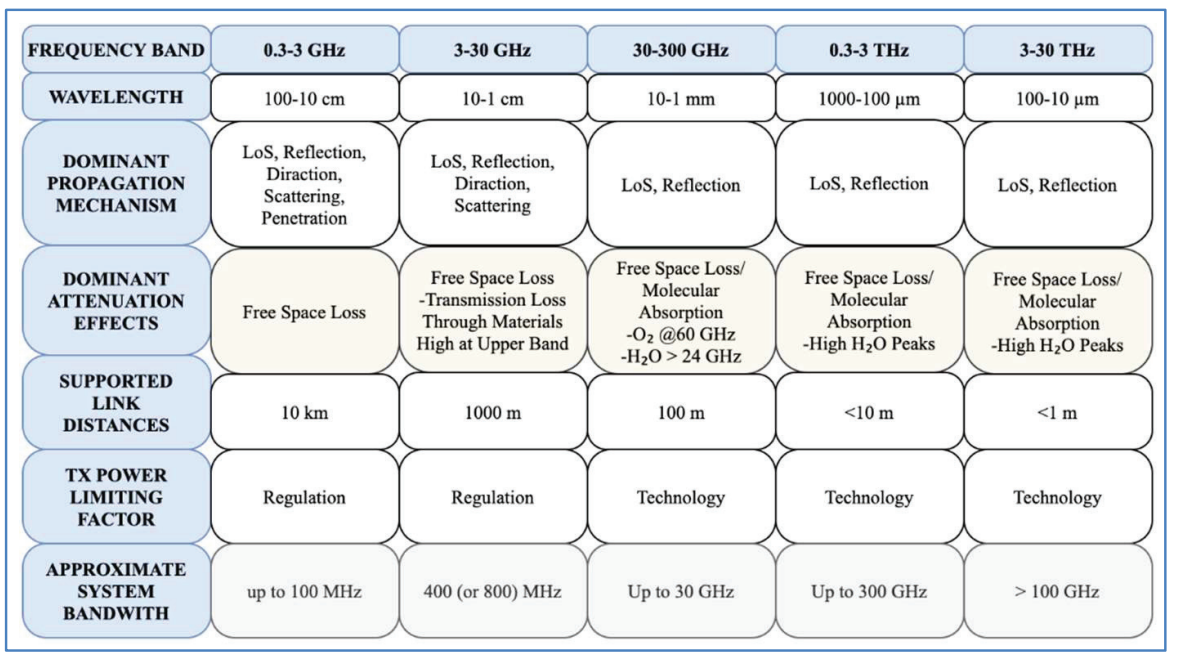

Figure 2 Characteristics of the spectrum bands for 5G and 6G [14].

and low latency communication. 6G aims to transform the world into a global digital village, as follows:

Human Digital Twin - With a collaboration of interdisciplinary sciences and $6 \mathrm{G}$ technology digital twins, the human body can be designed to form a virtual human world that realizes the human health data, monitoring in realtime. $6 \mathrm{G}$ will accurately associate urine biochemistry, blood routines, color Doppler ultrasound, and many other examinations with delivering a person his/her accurate or statement of their health status with time.

High-Speed Internet Access in the Air - 6G will be using new network strategies to support air users and will consider satellite transmission to enable high quality service provision (but expectedly with higher cost).

New Smart City - 6G will enable unified network architecture while adding new business scenarios. In $6 \mathrm{G}$ systems, IA will be deeply integrated and considered at multiple levels. for efficient transmission, internal security, seamless networking, large-scale deployment, automatic maintenance, and alarming hazards. Moreover, many sensors will be mounted over the city and on the buildings. In these environments, AI will enable different required processes without human intervention.

Cyber Robots and Autonomous Systems - 6G will boost the proliferation of autonomous systems and robots. $6 \mathrm{G}$ will promote a very high-level 
self-driving cars and will make life easy for travelers; the possibility of the self-driving car needs many efficient 6G-enable sensors, like odometer, inertial measurement equipment, sonar light detection, and many other. Furthermore, $6 \mathrm{G}$ will communicate between the UAVs and ground controllers. High demand applications in agriculture, science, business, military, logistics, aerial photography, disaster relief, and entertainment will be supported by swarms of UAVs.

XR (Extended Reality) is based on Holographic Communication - With the high-speed increase in the advancement of technology, expected in 2030, the need for virtual interaction will advance the Augmented Reality (AR)/VR to extended reality (XR). The customers will be allowed to enjoy the upgrade conveyed about by holographic communication and holographic display anytime and anywhere. XR will enable hearing, smell, touch, taste, sight and emotions, concerts, sports, painting, and other fully immersive holographic experiences.

Global Emergency Communication Rescue - 6G networks will be able to provide coverage in all regions by supporting different applications in emergency communications. For example, when earthquakes happen, the destruction of the terrestrial communication network can be covered with deployed unmanned aerial networks and satellite communications.

Smart Factory Plus - 6G networks will not be limited to only work in the factory. Instead, they will assure full connection and stability, security to the entire production cycle. $6 \mathrm{G}$ networks will quickly and flexibly connect intelligent devices that require to be connected inside the factory, and will provide dynamic adjustment according to the requirement of the production line using AI. Through the $6 \mathrm{G}$ network, Smart Factory PLUS will add an end-to-end closed loop.

It is expected that the $6 \mathrm{G}$ ecosystem creates opportunities for new businesses by implementing their use cases in real-time. According to the Finnish 6G Flagship [13], these innovations can enhance usage in a very innovative way and will pave the way to:

- Co-creation, bringing researchers and companies together;

- Research to business process, presenting research outputs to companies;

- Promoting research findings in relevant verticals/application areas while different players become forerunners in digitalization. 


\section{Enabling Technologies}

Technologies that enable $6 \mathrm{G}$ use cases contain, above $6 \mathrm{GHz}$, edge AI, communication with large, intelligent surfaces, integrated terrestrial, airborne and satellite networks, wireless transfer, and RF energy harvesting, terahertz communications, cell-free communication, artificial intelligence and carrier aggregation, by implementing multi-band scheduling, as follows:

Carrier Aggregation: Carrier aggregation (CA) can improve coverage, throughput, resource reuse, system capacity, fairness, service quality, and user experience. Telecommunication service providers provide high data rate application services, offering high service quality energy-efficient and costeffective. Consequently, mobile applications will meet the customer requirements through advanced methods for carrier aggregation, one of the main contributors to fulfill these requirements. Whereas CA has been successfully in (up to) Release 10 LTE-Advanced macro-cellular networks with typical bandwidths for each carrier of $20 \mathrm{MHz}$ (and up to $100 \mathrm{MHz}$, if available), and with up to five different non-contiguous carriers, bandwidths of hundreds of $\mathrm{MHz}$ for each carrier, up to 32 different carriers can be supported with Release 13 and beyond [16]. This enhanced framework brings augmented flexibility to aggregate a large number of carriers in different bands and will also be helpful for Licensed Authorized Access (LAA) operation in the unlicensed spectrum where large blocks of spectrum are available.

Communication with Large Intelligent Surfaces: For enabling better spectral and energy efficiency, Multiple-Input and Multiple-Output (MIMO) will be added to the 6G, MIMO, in the direction of large, intelligent surfaces and innovative environments, will support massive wireless communications surfaces $[6,17]$.

Wireless Power Transfer and Energy Harvesting: With the current trend of research on wireless energy, wireless energy transfer is getting mature. It is foreseen in $6 \mathrm{G}$ that base stations will provide essential power transfer for user devices. RF energy harvesting enables to get power from a nearby environment using RF communications, and provides an attractive solution for lifetime power-constrained users' batteries and their recharge. According to [18], the harvesting of energy from surrounding or specialized energy sources (low-power, energy-efficient wireless devices) can be self-sufficient and environment-friendly.

Communication in the Terahertz Band: The $\mathrm{THz}$ band will play a significant role in $6 \mathrm{G}$. It ranges from $0.1 \mathrm{THz}$ to $10 \mathrm{THz}$ and will provide 
more bandwidth, higher capacity, secure transmission, high energy efficiency, miniature transceivers, terabit-per-second link capacities, and ultra-high data rates [15].

Cell-Free Communication: Before 6G, the use of UAVs in a place with no infrastructure was assumed while, in $6 \mathrm{G}$, it is planned that they will be using it for the dead zone, also allowing cell-free communication. In $6 \mathrm{G}$, user equipments will be connected to the cell and the whole network. As a consequence, handover and coverage issues will be sorted out in new efficient ways.

Artificial Intelligence (AI) and Machine Learning (ML): For automation purposes, the AI and ML will fully support 6G. It will take the responsibilities of network selection, resource allocation, and handover, particularly in delay sensitive applications and to aid positioning.

Integrated Terrestrial, Airborne, and Satellite Networks: Drones will overcome terrestrial networks by supporting the hotspots connectivity. Further terrestrial and drones base stations will be in contact with satellite connectivity for better coverage availability. This type of communication aims to enhance user throughput and improve the services, considering the combination of high-altitude platforms, terrestrial base stations, and satellite stations.

\section{Standards and Standardization Bodies}

The outcome of any research is based on the set standards for its operation. It is defined as the requirements and specifications for a product, process, or service [19]. It is also defined as the accepted pattern with considered quality assurance. Furthermore, it is a set of regulations that directs its usage. In literal terms, standards are the way to do things to provide many benefits to one and all.

The need for standards is growing in hand with development in technology. The need for global information and communication technology (ICT) standards are evolving every day with the advancements. It is a prime necessity to have a set of standards to guide the entire development process to maintain interoperability among its processors and successors. The ICT standardization process for telecommunication networks is itself vast and critical $[20,21]$. The software and hardware companies are liable to comply with the interoperability standards within the multi-vendor ecosystem. 
Products must be standardized before they get into the commercial market. Standards are developed by organizations, alliances, or forums, i.e., Standards Development Organizations (SDOs).

\subsection{Standards Development Organizations (SDOs)}

SDOs are organizations, alliances, or forums that develop standards and regulations and are formed by stakeholders from different verticals, like manufacturers, providers, consumers, and regulators. Academia and industries play a vital role in setting out requirements and providing convincing arguments to develop a standard [19-21]. They also led down a set of regulations to ensure a legitimate development process. They also led down a set of regulations to ensure a legitimate development process. There are different types of SDOs based on different classifications. Figure 3 illustrates the SDOs classification and types, according to the European Telecommunications Standards Institute (ETSI) [21].

International SDOs represent the members globally and are members from National or Regional standard bodies, like ISO (International Organization for Standardization), IEC (International Electrotechnical Commission), ITU (International Telecommunication Union), among other. Regional SDOs include industry, academia, and national SDOs from various countries, like the African Organization for Standardization, formerly ARSO. In addition, at a country level, there are National SDOs (NSDOs), which issue

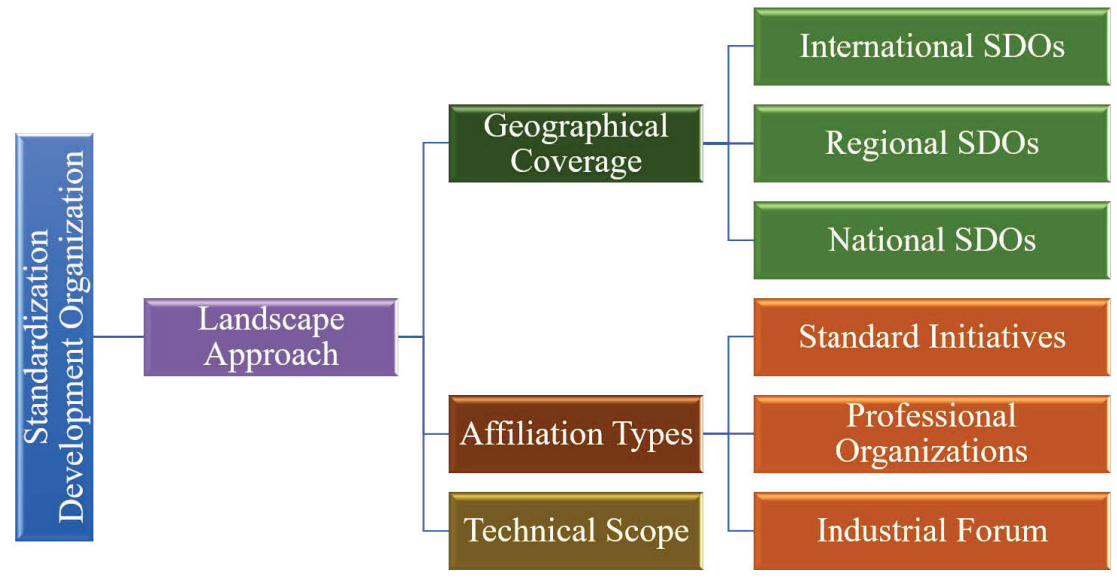

Figure 3 Types of standardization organizations. 
country-specific standards and collaborate with International and Regional SDOs [20].

SDOs set up Standard Initiatives groups to collaborate and coordinate standardization efforts on different subjects, like the 3rd Generation Partnership Project (3GPP) and oneM2M. Professional Organizations (POs) connect independent professionals to promote best practices and innovation in routine activities. The Internet Engineering Task Force (IETF) and the Institute of Electrical and Electronics Engineers Standards Association (IEEE-SA) are examples of POs.

\subsection{Standardization and Innovation}

The introduction of standards and requirements at the early stage of Research and Development (R\&D) activities is essential to avoid delays and identify the fallbacks. Standardization and research activities reside in a closely weaved structure and are developed in parallel. Depending on the landscape, standards play crucial parts in the entire development cycle. Innovation activities require that a pre-standard exists to guide the applied research. The ETSI Industry Standardization Groups (ISGs), for example, provide pre-standards to support research before achieving formal standards [20, 22]. Abided by the legal and regulatory obligations, standards define safety, safe practices, security and privacy procedures, among other.

\section{Spectrum Requirements for $6 \mathrm{G}$}

In 2019, the Federal Communications Commission (FCC) [23] opened a new spectrum range to boost $6 \mathrm{G}$ research activities. It has allowed frequencies between $95 \mathrm{GHz}$ and $3 \mathrm{THz}$ for research purposes and labelled it "the far frontier of spectrum policy." As 6G is going towards such high bands spectrum while operating in the ultra-high radio frequencies, enormous challenges arise in terms of propagation. Somehow, coexistence is of crucial importance for access to the frequency spectrum. Furthermore, some of the technologies as described below will be critical in 6G Networks.

\subsection{Spectrum Associated with 5G Networks}

The three megatrends established by ITU in IMT 2020 are Enhanced Mobile Broadband (eMBB), Massive Machine Type Communications (mMTC), and Ultra-Reliable and Low Latency Communications (URLLC). eMBB 
Table $15 \mathrm{G}$ spectrum allocation

\begin{tabular}{lcc}
\hline Frequency Bands & Range & Application \\
\hline Low bands & $<2 \mathrm{GHz}$ & eMBB, URLLC \& mMTC applications \\
Mid bands & $2-8 \mathrm{GHz}$ & \\
High bands & $>24 \mathrm{GHz}$ & eMBB and URLLC applications \\
\hline
\end{tabular}

describes the category for bandwidth-hungry applications such as highdefinition telepresence, telemedicine, remote surgery, and similar. In addition, it covers technologies like Carrier Aggregation (CA), Massive Input massive output (MIMO) or Multi RAT. mMTC describes the fast-growing, highvolume, dense IoT nodes/applications, such as smart metering, smart cities or asset tracking. These are mainly applications with Low Power, Low Cost, and Low Complexity requirements. Finally, URLLC includes mission-critical services such as autonomous vehicles, healthcare, and industrial automation and these applications strike for Low Latency, String Security, and Ultra Reliability.

In $5 \mathrm{G}$, the spectrum is allocated based on the applications described in Table 1 [12]. Since spectrum is a scarce resource, its availability is a challenge. Therefore, reusing the existing spectrum bands is essential in $6 \mathrm{G}$ to meet high bandwidth demands. The standardization initiatives to exploit the licensed and unlicensed spectrum will certainly be of fundamental importance in the coming years.

\subsection{New Spectrum Beyond THz}

New spectral bands need to be investigated in close collaboration with the terrestrial counterpart for propagation conditions and development of channel models encountered by the NTN (Non-Terrestrial Networks) elements both in inter-node communications and in non-terrestrial to terrestrial communications [20, 24]. In association with the 6G ecosystem for terrestrial applications, additional spectrum above $100 \mathrm{GHz}$ and NTN, the frequency bands beyond $50 \mathrm{GHz}$, and even above $\mathrm{THz}$ are expected to be used. In addition to that, NTN systems building on free-space optical communication can use even higher frequency bands (i.e., 150-300 THz). The architecture for evolutionary NTN systems will provide a cost-efficient network configuration by supporting flexible topologies and reusable infrastructure between terrestrial and non-terrestrial networks. UAVs can act as flying nodes of the $6 \mathrm{G}$ network capable of processing, computing, and communication functionalities. 


\subsection{Associated Challenges}

There is a vast difference between how devices operate in homogeneous and heterogeneous networks. The homogeneous networks support licensed bands wherein different technologies operating in unlicensed bands coexist. Some of the key challenges and design considerations are the following ones:

- Efficient spectrum sharing algorithm for complex network environments.

- Random motions of UEs and the frequent handovers in the dense environment influence spectrum sharing performance.

- 5G/6G support of reliable data transmission of varying sizes. Thus, the spectrum sharing strategy needs to support time-varying data requirements.

- Design collaboration algorithms among multiple Base Stations (BSs) and Access Points (APs) in heterogeneous networks.

\section{6G Standardization}

The world is moving towards the Beyond $5 \mathrm{G}$ communications ecosystem, supported by massive innovation and research activities. Different study groups are drafting an initial set of standards based on different use-cases and KPIs. 3GPP is expecting to publish 6G requirements beyond 2023 [7, 20]. Thus, fully-fledged $6 \mathrm{G}$ systems will mature in about a decade from now. Both academia and industry are working on a plethora of technologies like millimeter wavebands ( $\mathrm{mmW})$, terahertz $(\mathrm{THz})$ communications, RISs, incorporation of Artificial Intelligence (AI) at core design, ML at the radio interface, or holographic communications. In May 2019, ITU introduced IMT 2030 concept to define B5G as a hybrid network.

Industry giants, like AT\&T Inc., Facebook Inc., and QUALCOMM Inc., are working together in both $\mathrm{R} \& \mathrm{D}$ and deployment sectors towards $6 \mathrm{G}$ standards. On the other hand, the Next G Alliance [20, 24], formed in 2020, is working towards a $6 \mathrm{G}$ rollout from a different perspective, including deployment, R\&D, and manufacturing standardization. In the east, China formed alliances to initiate $6 \mathrm{G}$ early research [22].

In Europe, the European Commission Radio Spectrum Policy Group (RSPG) [25] recommends supporting, 6G initiatives by the European Commission (EC) and the Member States. It opens up for the usage of the European Union (EU) harmonized spectrum, as well as the flexible usage of new $\mathrm{THz}$ harmonized spectrum to support fixed wireless access and wireless 
backhauling $[22,25]$. The $6 \mathrm{G}$ Flagship project, centered at the University of Oulu of Finland, works on 6G technologies [13]. According to [26], 6G development should facilitate open collaboration and standardization between different stakeholders in a multi-disciplinary manner, ultimately creating true partnerships for all benefits. While pushing many technological boundaries on the journey to 6G, the UN Sustainable Development Goals (UN SDGs) are an essential lens to help prioritize development. Technology standards that support those use cases that hold the highest promise for improving human lives and protecting the environment need to be advanced first (by helping policymakers and involving communities). Vodafone Germany [27] announced a 6G research and development facility in Dresden. The EUfunded "Hexa-X" project, led by Nokia, is investigating future technologies for $6 \mathrm{G}$.

Japan [22] NTT Docomo is investing considerable funds to build a 6G research infrastructure, and they target to have the $6 \mathrm{G}$ specifications ready by 2025 . In South-Korea, Samsung has demonstrated an end-to-end 140 $\mathrm{GHz}$ wireless link using a fully digital beamforming solution in the $\mathrm{THz}$ spectrum [28] to mark its presence in $6 \mathrm{G}$ initiatives [15]. The R\&D institute NIIR [29] agreed to test 5G technology with Ericsson while collaborating with the Skolkovo Institute of Science and Technology [22] for 6G R\&D.

It is anticipated that 3 GPP Release 18 will bring new features and capabilities to 5G. Release 18 is expected to be approved by 2023. The following releases are expected to focus on $\mathrm{B} 5 \mathrm{G}$ and $6 \mathrm{G}$ specifications. The roadmap for $5 \mathrm{G}$ NR NTNs is expected to have products available with the finalization of 3GPP Rel. 17, and by 2023, 5G NR NTN systems will be operational. By 2025, new frequency bands, i.e., $>50 \mathrm{GHz}$ and beyond $\mathrm{THz}$, will be applicable for NTN systems. Figure 4 illustrates the overall roadmap for $6 \mathrm{G}$ development [20].

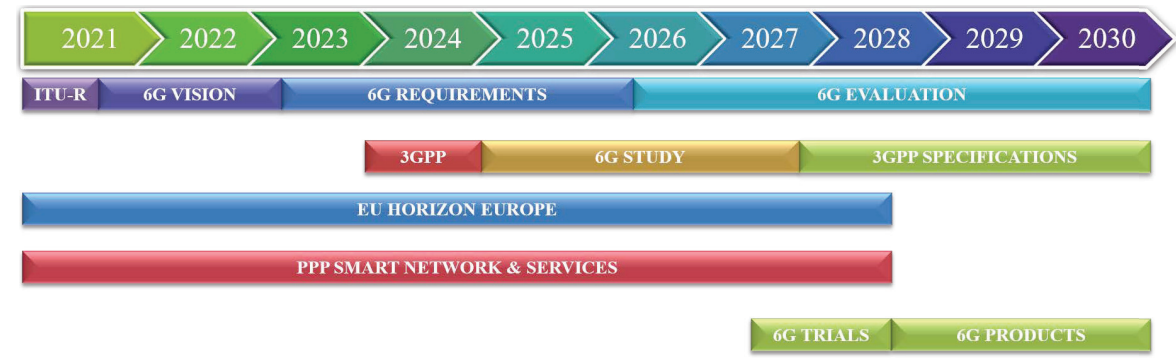

Figure 4 European union 6G development roadmap. 


\section{Conclusions}

This study has presented an overview of the standardization process and emphasized 6G standardization requirements while considering the challenges of spectrum usage in upper frequency bands. From a technology perspective, $6 \mathrm{G}$ will provide a vast opportunity to the users and MNOs to explore different services and use cases. New features of the 6G networks have been presented along with associated services and enabling technologies. After establishing newly evolved KPIs and requirements, various 6G standardization initiatives across the world have also been addressed. We have learned that the scope of standards and technology development needs to be broadened to support future ecosystems. Products need to comply with the standards to support the full capabilities of upcoming networks. Higher layers are made available for research which has brought new challenges along with enormous opportunities. Different organizations draft the specifications and standardization requirements for $6 \mathrm{G}$ in different use case scenarios and consider 6G guidelines, 100x data throughput, and sub-millisecond latency, compared to the $5 \mathrm{G}$ networks.

Consequently, the path towards the development of future wireless standards and technologies will be compelling. Critical considerations for the development of the next generation of standards and technologies are as follows. First, the SDOs need to increase their scope to bring new horizons to the communication ecosystem. Second, there is a need for the New Standards Evolution Paradigm to support reusability and scalability. Third, to overcome shortcomings in the SDOs, there is a need for Universal global standards to prevent their duplication. Finally, industry and markets should encourage the healthy and competitive development of the new and existing solution providers while ensuring a stable and sustainable global supply chain, without significant disruptions.

\section{Acknowledgment}

This work was supported by FCT/MCTES through national funds and when applicable cofounded EU funds under the project UIDB/50008/2020, ORCIP (22141-01/SAICT/2016) and TeamUp5G. TeamUp5G has received funding from the European Union's Horizon 2020 research and innovation programme under the Marie Skłodowska-Curie ETN TeamUp5G, grant agreement No. 813391. 


\section{References}

[1] I.-I. T. Union, "Report on Climate Change," ITU, 2008.

[2] G. Koutitas and P. Demestichas, "A review of energy efficiency in telecommunication networks," Proc. In Telecomm. Forum (TELFOR), November, 2009.

[3] F. Times, "Gartner Report," 2007.

[4] I. Cerutti, L. Valcarenghi and P. Castoldi, "Designing power-efficient WDM ring networks," in ICST International Conference on Networks for Grid Applications, Athens, 2009.

[5] W.Vereecken and et al., "Energy Efficiency in thin client solutions," in ICST International Conference on Networks for Grid Applications, Athens, 2009.

[6] P. Henrique and R. Prasad, 6G The Road to the Future Wireless Technologies 2030, River Publishers, 2021.

[7] J. Haas, T. Pierce and E. Schutter, "Datacenter Design Guide," Greengrid Whitepaper, 2009.

[8] J. Peha, "Spectrum MAnagement Policy Options," IEEE Communications Surveys, vol. 1, no. 1, pp. 2-8, 1998.

[9] B. Hassan, S. Baig and M. Asif, "Key Technologies for Ultra-Reliable and Low-LAtency Communication," 6G IEEE Communications Standards Magazine, pp. 106-113, 2021.

[10] GSMA, "Spectrum Navigator," March 2021. [Online]. Available: https: //www.gsma.com/spectrum/wp-content/uploads/2021/03/Spectrum-Na vigator-Q1-2021.pdf. [Accessed September 2021].

[11] M. Matinmikko-Blue, S. Yrjölä and P. Ahokangas, "Spectrum Management in the 6G er: The role of regulation and spectrum sharing," in 2nd $6 G$ Wireless Summit, 2020.

[12] Nidhi, A. Mihovska and R. Prasad, "Spectrum Sharing and Dynamic Spectrum MAnagement Techniques in 5G and Beyond Networks: A survey," Journal of Mobile Multimedia, pp. 65-78, 2021.

[13] O. University, "6G Flagship," 12 July 2021. [Online]. Available: www. oulu.fi/6gflagship/. [Accessed 29 July 2021].

[14] M. Latva-aho and K. Leppanen, "Key drivers and research challenges for 6G ubiquitous wireless intelligence (white paper)," 6G Flagship, University of Oulu, Oulu, 2019.

[15] S. Elmeadawy and R. Shubair, "Enabling Technologies for 6G future wireless communications: Opportunities and Challenges," arXiv, 2020. 
[16] Z. Shen, A. Papasakellariou, J. Montojo, D. Gerstenberger and F. Xu, "Overview of 3GPP LTE-Advanced Carrier Aggregation for 4G Wireless Commnications," IEEE Communications Magazine, pp. 122-130, 2012.

[17] W. Saad, M. Bennis and M. Chen, "A vision of 6G wireless systems: Applications, trends, technologies and open research problems," IEEE Network, pp. 134-142, 2019.

[18] L. P. G. W. a. Y. Z. Yu Luo, "RF Energy Harvesting Wireless Communications: RF Environment, Device Hardware and Practical Issues," Sensors, 2019.

[19] A. Akins, "6G Wireless: What it is and when it's coming," March 2021. [Online]. Available: www.spglobal.com/marketintelligence/en/news-in sights/latest-news-headlines/6g-wireless-what-it-is-and-when-it-is-c oming-62956538. [Accessed 2707 2021].

[20] 5GPPP, "B5G/6G Research with Standarization Potential Roadmap," 5GPPP: 5G Infrastructure Public Private Partnership, November 2020.

[21] C.J. Lanting and A. Rodriguez-Ascaso, "ETSI: Understanding ICT Standardization: Principles and Practice," 2021. [Online]. Available: https://www.etsi.org/images/files/Education/Understanding_ICT_S tandardization_LoResWeb_20190524.pdf. [Accessed 28 July 2021].

[22] T. Spanjaard, "Who Will Standardize 6G? Smartinsights," Smartinsights www.smartinsights.net/single-post/who-will-standardize-6g, 11 December 2020.

[23] S. Kinney, "Looking beyond 5G, FCC Opens up Terahertz Spectrum," RCR Wireless News www.rcrwireless.com/20190319/policy/fcc-terahe rtz-spectrum, 19 March 2019.

[24] T. 5. I. Association, "5GPPP: European Vision for the 6G Network Ecosystem," 7 June 2021. [Online]. Available: 5g-ppp.eu/european-v ision-for-the-6g-network-ecosystem/. DOI: 10.5281/zenodo.5007671. [Accessed 29 July 2021].

[25] E. R. S. P. Group, "RSPG Additional spectrum needs and guidance on the fast rollout of future wireless broadband networks," March 2021. [Online]. Available: https://rspg-spectrum.eu/wp-content/uploads/2021 /02/RSPG21-008final_Draft_RSPG_Opin. [Accessed July 2021].

[26] S. A. M. I. A. H. B. Y. C. S. D. R. J. P. K. M. K. M. K. P. K. a. o. Marja Matinmikko-Blue, "White Paper on 6G Drivers and the UN SDGs," arXiv preprint arXiv:2004.14695, 2020.

[27] M. Lennighan, "Vodafone Germany Launches a Network Offensive," June 2021. [Online]. Available: https://Telecoms.com/, 26 May 2021, 
telecoms.com/509917/vodafone-germany-launches-a-network-offensiv e/. [Accessed July 2021].

[28] A. Hernandez, "Techaeris: "South Korea Eyes 2028 to LAUNCH 6G, Samsung Leads the Charge.,", 23 June 2021. [Online]. Available: tech aeris.com/2021/06/23/south-korea-eyes-2028-to-launch-6g-samsungleads-the-charge/. [Accessed 31 July 2021].

[29] Telecompaper, "Russian R\&D Institute NIIR to Test 5G with Ericsson," 2021. [Online]. Available: www.telecompaper.com/news/russian-rand d-institute-niir-to-test-5g-with-ericsson--1353665. [Accessed 2021].

\section{Biographies}

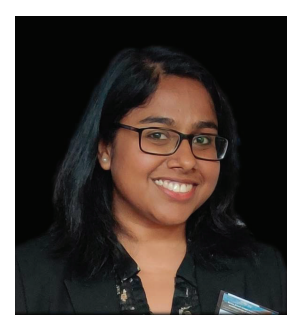

Nidhi is an Early-Stage Researcher in the project TeamUp5G, a European Training Network in the frame of (MSCA ITN) of the European Commission's Horizon 2020 framework. Currently enrolled as a $\mathrm{PhD}$ student at Aarhus University in the Department of Business Development and Technology. She received her Bachelor's degree in Electronics and Telecommunication and Masters' degree in Electronics and Communication (Wireless) from India. Her research interests are small cells, spectrum management, carrier aggregation, etc. 


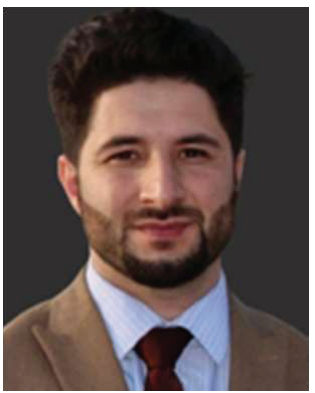

Bahram Khan is an Early-Stage Researcher since November 2019 at the Instituto de Telecomunicacoes and a Ph.D. student at the University of Beira Interior Covilhã, Portugal. He did his bachelor's degree from COMSATS University in 2016 and Master studies from Bahria University in 2018 in the department of Electrical Engineering from Pakistan Islamabad. His research interest is HetNet, small cell deployments, carrier aggregation, cell-free. Currently, the project on which he is working is focused on $5 \mathrm{G}$ and beyond, which is titled as "Licensed Shared Access in Heterogeneous Network with Small Cells" and is under the TeamUp5G and funded by Marie Skłodowska-Curie Innovative Training Networks (MSCA ITN) of the European Commission's Horizon 2020 framework.

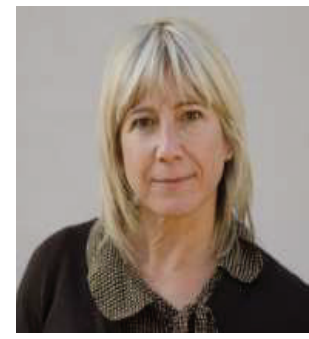

Albena Mihovska is an Associate Professor at the Department of Business Development and Technology, Aarhus BSS, Aarhus University, Denmark. She has been with the Department of Business Development and Technology, Aarhus BSS, Aarhus University since February 2017. She received her PhD in Mobile wireless communications, Aalborg University, Denmark. Albena Mihovska is currently teaching a 1st-semester course in the B.Sc. and GMM programs and is a supervisor of TS and master projects the Cand. Polyt program. 


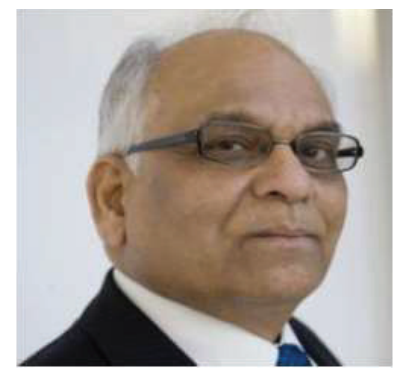

Ramjee Prasad, is a Professor of Future Technologies for Ecosystem Innovation, (FT4BI) in the department of Business Development and Technology, Aarhus University, Denmark. He is a Founder President of CTIF Global Capsule (CGC). He is also the Founding Chairman of the Global ICT Standardization Forum for India (GISFI: www.gisfi.org) established in 2009. GISFI has the purpose of increasing the collaboration between Indian, Japanese, European, North-American, Chinese, Korean and other worldwide standardization activities in the area of Information and Communication Technology (ICT) and related application areas. He is also a Fellow of IEEE, USA; IETE India; IET, UK; and a member of the Netherlands Electronics and Radio Society (NERG), and the Danish Engineering Society (IDA).

He was honoured by the University of Rome "Tor Vergata", Italy as a Distinguished Professor of the Department of Clinical Sciences and Translational Medicine on March 15, 2016. He is an Honorary Professor of the University of Cape Town, South Africa, and the University of KwaZuluNatal, South Africa. He received Ridderkorset af Dannebrogordenen (Knight of the Dannebrog) in 2010 from the Danish Queen for the internationalization of top-class telecommunication research and education.

He has received several international awards such as IEEE Communication Society Wireless Communication Technical Committee Recognition Award in 2003 for making a contribution in the field of "Personal, Wireless and Mobile System Networks", Telenor's Research Award in 2005 for impressive merits, both academic and organizational within the field of Wireless and Personal Communication, 2014 IEEE AESS Outstanding Organizational Leadership Award for Organizational Leadership in developing and globalizing the CTIF (Center for TeleInFrastruktur) Research Network, and so on.

He has been Projected Coordinator of several EC Projects, MAGNET, MAGNET Beyond, e-Wall and so on. He is the founding editor-in-chief of 
the Springer International Journal on Wireless Personal Communications. $\mathrm{He}$ is member of the editorial board of several other renowned international journals and is the series editor of the Artech House Universal Personal Communications Series. Ramjee Prasad is a member of the Steering, Advisory, and Technical Program committees of many renowned annual international conferences, e.g., Wireless Personal Multimedia Communications Symposium (WPMC); Wireless VITAE, etc. He has published more than 30 books, 1000 plus journals and conferences publications, more than 15 patents, a sizable number of graduated Ph.D. students (over 140) and an even larger number of graduated M.Sc. students (over 250). Several of his students are today worldwide telecommunication leaders themselves.

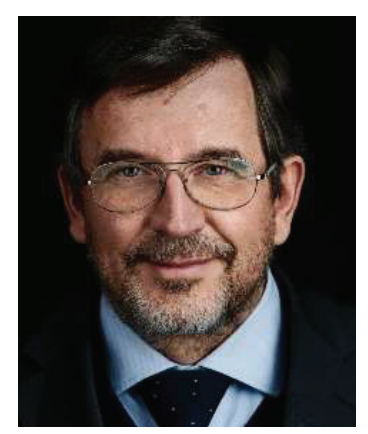

Fernando J. Velez (M93SM05) received the Licenciado, M.Sc., and Ph.D. degrees in Electrical and Computer Engineering from Instituto Superior Tecnico, the Technical University of Lisbon in 1993, 1996, and 2001, respectively. Since 1995 he has been with the Department of Electromechanical Engineering of Universidade da Beira Interior, Covilhã, Portugal, where he is Assistant Professor. He is also a senior researcher of Instituto de Telecomunicações. Fernando was an IEF Marie Curie Research Fellow in King's College London (KCL) in 2008/09 (OPTIMOBILE IEF) and a Marie Curie ERG fellow at Universidade da Beira Interior from 2010 until March 2013 (PLANOPTI ERG). Fernando is the coordinator of the Instituto de Telecomunicações team in the Marie Skłodowska-Curie ITN Action (TeamUp5G) that started in 2019. He made or makes part of the teams of several European and Portuguese research projects on mobile communications, and he was the coordinator of six Portuguese projects. Recently, he was the coordinator of CONQUEST (CMU/ECE/0030/2017), an exploratory 


\section{Nidhi et al.}

project from Carnegie Mellon University (CMU) Portugal, a collaboration with the Department of Engineering and Public Policy from CMU. He has authored three books, 24 book chapters, 160 papers and communications in international journals and conferences, plus 39 in national conferences.

Fernando is currently the IEEE VTS Region 8 (Europe, Middle East and Africa) Chapter Coordinator (nominated by VTS in 2010) and was the elected IEEE VTS Portugal Chapter coordinator from 2006 to 2014. He is also the adjunct coordinator of the Telecommunications Specialization of Ordem dos Engenheiros. Prof. Velez was also the coordinator of the WG2 (on Cognitive Radio/Software-Defined Radio Co-existence Studies) of COST IC0905 TERRA. His main research areas are cellular planning tools, traffic from mobility, wireless body sensor networks and wearable technologies, crosslayer design, spectrum sharing/aggregation, and cost/revenue performance of advanced mobile communication systems. 